\section{Sterile keratitis after combined riboflavin-UVA corneal collagen cross-linking for keratoconus}

FC Lam, P Geourgoudis, MA Nanavaty, $S$ Khan and D Lake

\begin{abstract}
Purpose To investigate patient risk factors and to look for potential causes of sterile infiltrates following an unexpected cluster of sterile keratitis after a routine collagen crosslinking (CXL) list.

Methods The records of all 148 cases

of CXL were reviewed retrospectively. The equipment and solutions used and our clinic's standard operating procedure for CXL were reviewed. An in-vitro experiment to explore the variation in ultraviolet A (UVA) irradiance from fluctuations in the working distance of the UVA lamp was conducted. Results The four patients who developed sterile infiltrates had steeper maximum corneal curvatures $(68.0 \pm 7.3 \mathrm{D})$ and thinner pachymetry $(389.9 \pm 49.0 \mu \mathrm{m})$ than the 144 who did not $(57.0 \pm 8.2 \mathrm{D}, P=0.05 ; 454.6 \pm 45.4 \mu \mathrm{m}$, $P=0.08$ ). A corneal curvature of $>60$ Dand a pachymetry of $<425 \mu \mathrm{m}$ were significant risk factors. All four affected cases obtained a complete resolution with topical antibiotics and steroids. The unaided VA and the maximum $K$ improved from their preoperative levels in three out of four patients. A 2-mm reduction in distance of the VEGA C.B.M. X-Linker from a treated surface increased irradiance to $3.5-3.7 \mathrm{~mW} / \mathrm{cm}^{2}$, which is above the threshold for endothelial toxicity. Conclusion Patients with thinner and steeper corneas are at an increased risk of developing sterile keratitis. The visual outcomes despite this complication are good. Eye (2014) 28, 1297-1303; doi:10.1038/eye.2014.173;

degenerative disorder of the cornea,

characterized by stromal thinning and conical ectasia, resulting in irregular astigmatism and associated visual loss. ${ }^{1}$ In 2003, Wollensak et $a l^{2}$ demonstrated in 23 eyes of 22 patients that the progression of keratoconus was stopped by corneal collagen cross-linking (CXL) with a reduction of the maximal keratometry readings by $2.01 \mathrm{D}$, a reduction in the refractive error by $1.14 \mathrm{D}$ in 16 eyes $(70 \%)$, and a slight improvement in visual acuity in 15 eyes (65\%). The efficacy of CXL as a treatment for progressive keratoconus was subsequently confirmed on further studies. ${ }^{3-5}$

Sterile infiltrates, all of which resolved within 4 weeks with treatment of dexamethasone four times a day, have been reported in $7.6 \%$ of eyes following CXL. ${ }^{6}$ Recently, four out of seven patients in a single routine CXL list at our clinic developed sterile infiltrates following uncomplicated procedures. Before this, we had treated 141 eyes over 4 years with corneal collagen cross-linking without complications. To date no clear risk factors or cause for the development of these sterile infiltrates has been found and the true etiology of these infiltrates is unknown, although several different authors have come up with different hypotheses for possible causes of these sterile infiltrates. ${ }^{7-11}$ As all the cases occurred on a single list at our clinic, this suggested that all the patients were probably exposed to the same predisposing factor. Therefore, we used this undesired event as a unique opportunity to investigate for factors that predispose to sterile infiltrates.
\end{abstract} published online 8 August 2014

\section{Introduction}

Keratoconus is a common condition affecting 1 in 2000 of the population. It is a non-inflammatory,

\section{Materials and methods}

A detailed review of the seven patients who received CXL on the same operating list was performed to look for possible pre-operative
The Corneoplastic Unit, Queen Victoria Hospital, West Sussex, UK

Correspondence: FC Lam, The Corneoplastic Unit, Queen Victoria Hospital, Holtye Road, West Sussex RH19 3DZ, UK Tel: +44 (0)1342 414 000; Fax: +44 (0)1342 414106. E-mail: fook_chang@ hotmail.com

Received: 22 March 2013 Accepted in revised form: 23 May 2014 Published online: 8 August 2014 
risk factors. The outcomes of the four patients with sterile keratitis were followed prospectively and the 3-month follow-up outcomes are presented. To further assess patient risk factors for developing sterile keratitis following $\mathrm{CXL}$, a retrospective review of the remaining preceding 141 consecutive cases of CXL at our clinic was also performed.

To evaluate intra-operative risk factors, the standard operating procedure and the ultraviolet A (UVA) lamp used for CXL in our clinic were reviewed. The susceptibility of the UVA lamp to fluctuations in working distance was also tested (see 'In-vitro Experiments on the UVA source for $\left(X L^{\prime}\right)$. The sterilization procedures for the equipment and the solutions used in the CXL process for the seven patients from when the cluster of four sterile keratitis cases developed were also examined.

\section{Assessments of patients undergoing CXL}

Prior to CXL, central corneal thickness measurements were obtained using ultrasound pachymetry with a SP100 pachymeter (Tomey, Nagoya, Japan) and the Pentacam (Oculus, Wetzlar, Germany) was used to obtain maximum $K$ readings and corneal thickness measurements at the pupil center of the patients' corneas. Endothelial cell counts were obtained with an EM3000 specular microscope (Tomey).

\section{Technique of CXL}

The CXL for all seven patients were performed by a single experienced surgeon (MAN) with the assistance of one nurse. Our technique of CXL, which is described in detail elsewhere, ${ }^{12}$ involves the disruption of the corneal epithelium with a Daya Epithelial Disruptor (Duckworth \& Kent, Baldock, UK). (See Supplementary File for the video of epithelial disruption). Corneal epithelium is not removed thereby preserving the thickness of the cornea thereby allowing the treatment of patients with cornea thicknesses down to $400 \mu \mathrm{m}$. Iso-osmolar riboflavin $0.1 \%$ solution with dextran 20\% T500 (Sooft Italia S.p.A, Montegiorgio, Italy) was applied every $5 \mathrm{~min}$ for the $30 \mathrm{~min}$ prior to and every $5 \mathrm{~min}$ for the $30 \mathrm{~min}$ during the irradiation of the patient's cornea with UVA at an irradiance of $3 \mathrm{~mW} / \mathrm{cm}^{2}$. Additional iso-osmolar riboflavin $0.1 \%$ drops were instilled when the pre-corneal film started to break up. At the end of the procedure, a bandage contact lens was placed for ocular comfort and patients were started on topical chloramphenicol QID. Topical steroids were not prescribed in the immediate post-operative period. The procedure itself was uncomplicated in all seven patients. However, four of the patients developed an infiltrate and significant inflammation of the eye with severe ocular discomfort and photophobia in the early post-operative period (that is, within the first week).

\section{In-vitro experiments on the UVA source for CXL}

The UVA lamp used in our clinic was the VEGA C.B.M. $\mathrm{X}$-Linker (CSO, Scandicci, Italy) which has a working distance of $54 \mathrm{~mm}$. Collimation is provided by a pair of red LEDs. We tested the tolerance of this UVA lamp to inadvertent changes in the working distance by varying the distance of the lamp by $1 \mathrm{~mm}$ increments and decrements, and measuring the irradiance from the lamp with a UVA-Light Meter (Lutron Electronic Enterprise Company Limited, Taipei, Taiwan).

\section{Statistical analysis}

All data was recorded onto an Excel spreadsheet and statistical analysis was performed on Excel. Tests for statistical significance between the four affected cases with the remaining three unaffected cases from the same list and between the four affected cases and the remaining 144 unaffected cases treated at our clinic over a 4-year period were done using a two-tailed unpaired $t$-test. Tests for statistical significance when comparing pre- against post-operative change was done with a two-tailed paired $t$-test. The two-tailed Fisher's exact test was used to assess the significance of maximum corneal curvatures and thinnest corneal pachymetry as risk factors for the development of sterile keratitis. To do this, cases were divided into cases with a corneal pachymetry of $<425 \mu \mathrm{m}$ and $>425 \mu \mathrm{m}$, or cases with a maximum corneal curvature of $<60 \mathrm{D}$ and $>60 \mathrm{D}$. A $P$-value of $\leq 0.05$ was taken to be significant.

\section{Results}

CXL procedures (148) were performed at our clinic in a 4-year period. The four cases of sterile keratitis emanated from an operating list of seven patients (Table 1). All seven cases were receiving CXL for documented progression in their keratoconus. The mean age of the four patients who developed sterile keratitis was 26.0 years (SD: 8.8 years); while the mean age of the other three patients on the operating list who did not develop sterile keratitis was 21.3 years (SD: 6.6 years; $P=0.49$ ) and the mean age of the remaining 144 cases was 27.6 years (SD: 10.8 years; $P=0.73)$.

The maximum corneal curvatures for the four cases who developed the infiltrate were on average 68.0 D (SD: 7.3 D; Figure 1 and Table 1); while the maximum corneal curvatures of the remaining three unaffected cases on the 
Table 1 Pre-operative characteristics of the seven patients presenting for CXL

\begin{tabular}{|c|c|c|c|c|c|c|c|}
\hline Patient & 1 & 2 & 3 & 4 & 5 & 6 & 7 \\
\hline Post-operative infiltrate & \multicolumn{4}{|c|}{ Yes } & \multicolumn{3}{|c|}{ No } \\
\hline Age (years) & 21 & 23 & 39 & 20 & 29 & 17 & 18 \\
\hline Sex & Male & Male & Male & Male & Female & Male & Female \\
\hline \multicolumn{8}{|l|}{ Pre-operative corneal thickness $(\mu \mathrm{m})$} \\
\hline Ultrasound & 467 & Not done & 567 & 423 & 521 & 491 & 521 \\
\hline Pupil center on pentacam & 417 & 434 & 451 & 447 & 446 & 486 & 501 \\
\hline Cone apex on pentacam & 418 & 436 & 378 & 425 & 438 & 461 & 504 \\
\hline Thinnest location on pentacam & 405 & 422 & 317 & 415 & 411 & 461 & 497 \\
\hline Pre-operative maximum K (D) & 66.1 & 66 & 78.4 & 61.4 & 57.4 & 46.3 & 57.4 \\
\hline Refractive spherical equivalent (D) & -14.5 & -4.9 & -12.4 & -1.75 & -5.4 & -1 & -5.375 \\
\hline Refractive astigmatism (D) & -15 & -3.75 & -4.75 & -2 & -6.25 & -1 & -6.25 \\
\hline Pre-operative endothelial cell count (standard deviation) & $1093(709)$ & Unable & $2952(186)$ & 2977 (105) & $2676(374)$ & 2615 (195) & $2676(171)$ \\
\hline
\end{tabular}

Abbreviation: CXL, collagen cross-linking.
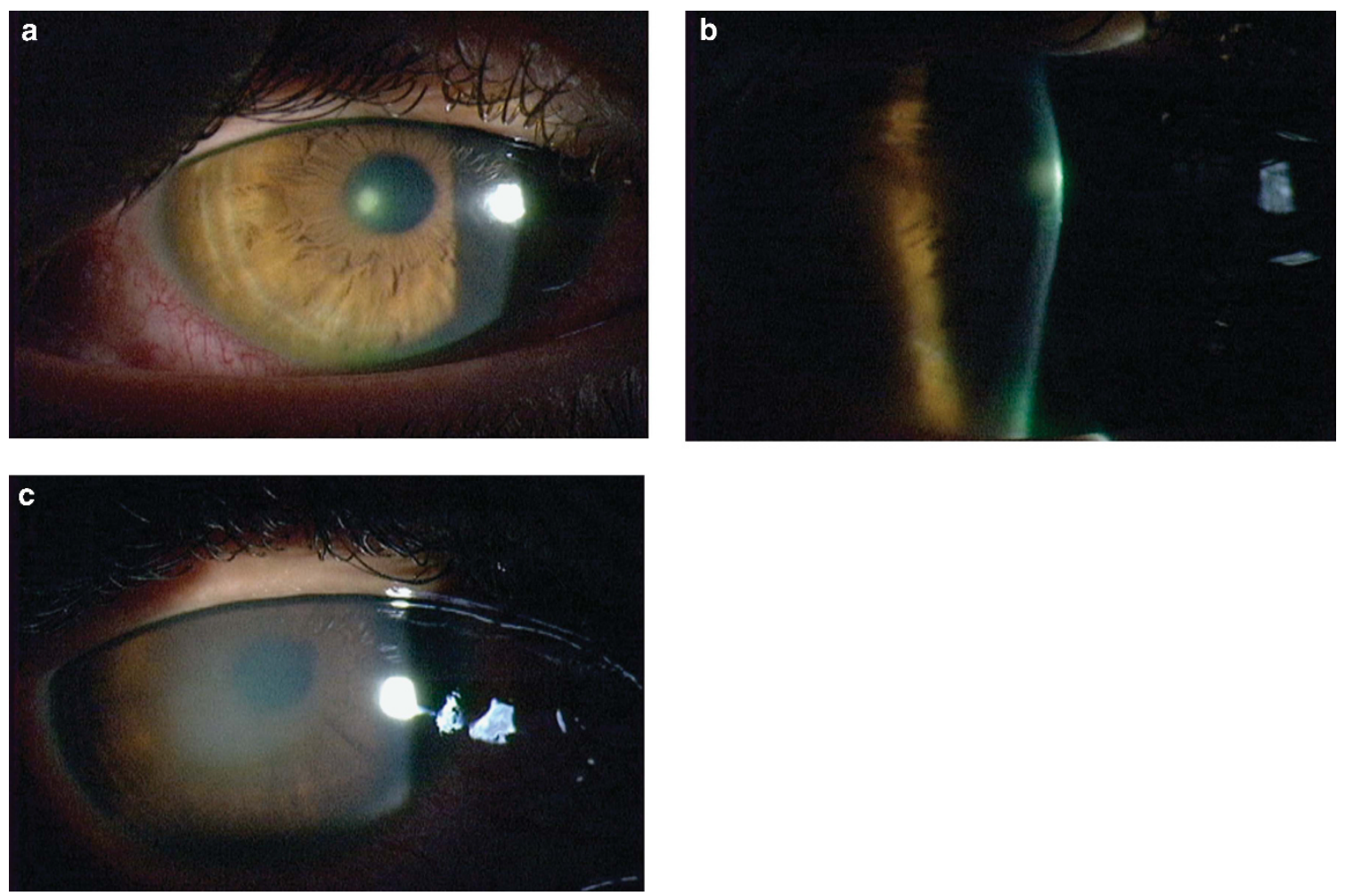

Figure 1 Post-operative slit lamp photographs demonstrating the post-CXL sterile keratitis. (a) Patient 1—sterile infiltrate. (b) Patient 1 -optical cross-section of sterile infiltrate. (c) Patient 2-resolving sterile infiltrate.

same operating list was $53.7 \mathrm{D}$ (SD: $6.4 \mathrm{D} ; P=0.04)$. The maximum corneal curvatures of the 144 consecutive unaffected cases were also on an average lower than the four affected cases at 57.0 D (SD: 8.2 D; $P=0.05)$. Of the 148 cases treated at our clinic, 38 cases had a maximum corneal curvature of $>60 \mathrm{D}$ but only four of these cases (10.5\%) developed a corneal infiltrate, while none of the remaining 110 cases who had a maximum corneal curvature of $<60 \mathrm{D}$ developed a corneal infiltrate $(P=0.0038$, Fisher's exact test $)$.
The thinnest corneal pachymetry on Pentacam for the four cases who developed corneal infiltrates was on an average $389.9 \mu \mathrm{m}$ (SD: $49.0 \mu \mathrm{m}$ ), while the thinnest corneal pachymetry for the other three remaining unaffected cases on the same list was $456.3 \mu \mathrm{m}$ (SD: $43.2 \mu \mathrm{m} ; P=0.12$ ) and the thinnest corneal pachymetry for the 144 unaffected cases was on an average $454.6 \mu \mathrm{m}$ (SD: $45.4 \mu \mathrm{m} ; P=0.08$ ). Of the 148 cases treated at our clinic, 24 cases had a thinnest corneal pachymetry of $<425 \mu \mathrm{m}$ but only four of these cases (16.7\%) developed 
a corneal infiltrate, while none of the remaining 124 cases with a thinnest corneal pachymetry of $>425 \mu \mathrm{m}$ developed a corneal infiltrate $(P=0.0006$, Fisher's exact test).

Fifteen of the 148 cases treated at our clinic had a maximum corneal curvature of $>60 \mathrm{D}$ and a corneal pachymetry of $<425 \mu \mathrm{m}$, and four of these patients (26.7\%) developed sterile keratitis. None of the remaining 133 cases with a corneal pachymetry of $<60 \mathrm{D}$ and a thinnest corneal pachymetry of $>425 \mu \mathrm{m}$ developed a corneal infiltrate. This co-existence of a maximum corneal curvature of $>60$ Dand a thinnest corneal pachymetry of $<425 \mu \mathrm{m}$ was a statistically significant risk factor ( $P=0.001$, Fisher's exact test).

All patients developed the corneal infiltrates within the first post-operative week. The corneal infiltrates occurred in the thinnest area of the patients' corneas and at the level of the anterior to mid corneal stroma. There was only one infiltrate in three cases (Figure 1), while there were two infiltrates in patient 3 . Patient 1 appeared to have a circular endothelial plaque. A small epithelial defect was present in patient 1 and 2, and a mucous plaque was present in patient 3; while patient $4 \mathrm{did}$ not have an epithelial defect. In addition, in all four cases there was circumcorneal injection and varying degrees of corneal edema and Descemet folds but there was no anterior chamber inflammation.

\section{Post-operative course and management of affected patients}

All four patients were treated as cases of possible infective keratitis. Corneal scrapes were taken for gram stains and for culture. All four patients were started on hourly topical ofloxacin. There was no significant improvement on this treatment and the patients were started on guttae dexamethasone $0.1 \%$ minims (Bausch \&
Lomb, Rochester, NY, USA) after 2 days with a rapid resolution of symptoms and signs. There were no organisms seen on the gram stains of all four patients and there was no growth from the cultures from all four patients. All four patients had settled with a complete resolution of the infiltrate within 1 month. However, a persistent stromal haze was noted in one patient.

All four patients have completed a 3-month follow-up period. The pre- and post-operative visual acuities, maximum keratometry readings, refractions, and endothelial cell counts are outlined in Table 2. However, the quality of the endothelial cell count in patient 1 was poor and is likely to be inaccurate, as reflected by the high standard deviation of the endothelial cell count. The patients unaided visual acuities improved in two patients, remained the same in one patient, and reduced in one patient, although the pinhole visual acuity of this patient remained the same. The maximum $K$ readings reduced in three out of the four affected patients. The average maximum $K$ reduced from $68.0 \mathrm{D}(\mathrm{SD}=7.3 \mathrm{D})$ to $66.2 \mathrm{D}$ $(\mathrm{SD}=8.6 \mathrm{D} ; P=0.12$, paired $t$-test). The mean endothelial cell count in the affected patients was 2340 cells $/ \mathrm{mm}^{2}$ $(\mathrm{SD}=1080.6)$ pre-operatively but 2736 cells $/ \mathrm{mm}^{2}$ $(\mathrm{SD}=180.0) 3$ months post-operatively, while the median endothelial cell counts were 2952 cells $/ \mathrm{mm}^{2}$ and 2785 cells / $\mathrm{mm}^{2}$. There was no statistical significant difference between the pre-operative and 3-month post-operative average endothelial cell counts $(P=0.63$, paired $t$-test).

\section{Assessment of CXL equipment, solutions, and standard operating procedure}

The VEGA C.B.M. X-Linker was checked and serviced by an engineer from the suppliers. The VEGA C.B.M.

$X$-Linker was in good working order with no defective parts. From an evaluation of the theatre logbooks, we

Table 2 Pre- and post-operative visual acuities, maximum Ks and endothelial cell densities of patients who developed infiltrate post-C3R

\begin{tabular}{|c|c|c|c|c|c|c|}
\hline Case & $\begin{array}{l}\text { Pre-operative } \\
\text { visual acuity }\end{array}$ & $\begin{array}{l}\text { Post-operative } \\
\text { visual acuity }\end{array}$ & $\begin{array}{l}\text { Pre-operative } \\
\text { maximum } \mathrm{K}\end{array}$ & $\begin{array}{l}\text { Maximum } \mathrm{K} \text { at } \\
\text { last follow-up }\end{array}$ & $\begin{array}{l}\text { Pre-operative endothelial cell } \\
\text { count (standard deviation) }\end{array}$ & $\begin{array}{l}\text { Post-operative endothelial cell } \\
\text { count (standard deviation) }\end{array}$ \\
\hline 1 & $\begin{array}{l}\text { 20/100 unaided } \\
\text { 20/40 BSCVA } \\
\text { 20/40 pinhole }\end{array}$ & $\begin{array}{l}\text { 20/80 unaided } \\
20 / 50 \text { pinhole }\end{array}$ & 66.1 & 63.8 & 1093 (709) & 2887 (147) \\
\hline 2 & $\begin{array}{l}\text { 20/200 unaided } \\
\text { 20/100 BSCVA } \\
\text { 20/60 pinhole }\end{array}$ & $\begin{array}{l}\text { 20/160 unaided } \\
20 / 30 \text { pinhole }\end{array}$ & 66.0 & 62.3 & Unable & Unable \\
\hline 3 & $\begin{array}{l}\text { 20/200 unaided } \\
\text { 20/125 BSCVA, } \\
\text { 20/30 CL, } \\
20 / 30 \text { pinhole }\end{array}$ & $\begin{array}{l}10 / 200 \text { unaided } \\
20 / 30 \text { pinhole }\end{array}$ & 78.4 & 78.8 & 2952 (186) & 2785 (214) \\
\hline 4 & $\begin{array}{l}\text { 20/63 unaided, } \\
\text { 20/50 BSCVA, } \\
\text { 20/50 pinhole }\end{array}$ & $\begin{array}{l}20 / 63 \text { unaided } \\
20 / 25 \text { pinhole }\end{array}$ & 61.4 & 59.7 & 2977 (120) & $2537(120)$ \\
\hline
\end{tabular}

Abbreviations: BSCVA=best spectacle corrected visual acuity; $C L=$ contact lens. 
were able to confirm that the irradiance of the lamp had been checked before each case and the irradiance had been found to be at $\sim 3 \mathrm{~mW} / \mathrm{cm}^{2}$ at the working distance of $54 \mathrm{~mm}$.

From our own assessment of the X-linker, we found that at the specified working distance of $54 \mathrm{~mm}$, the radiated power density was $\sim 3 \mathrm{~mW} / \mathrm{cm}^{2}$. Decreasing the distance between the UVA lamp and the surface being treated resulted in the irradiance increasing but increasing the distance between the UVA lamp and the surface being treated resulted in the irradiance decreasing (Figure 2). If the lamp was brought forward by even just $2 \mathrm{~mm}$, the irradiance increased to between 3.5 and $3.7 \mathrm{~mW} / \mathrm{cm}^{2}$.

There was no particular order in which patients on the CXL list were affected by the corneal infiltrates. From this, we were able to establish that affected patients had received riboflavin drops from different manufactured batches with different batch numbers. The instruments used in CXL also came from different sterilization batches and we were also able to exclude this as a possible cause of the corneal infiltrates.

\section{Discussion}

Transient intra-stromal corneal opacities have been reported following CXL. In these cases the infiltrates have been multiple, situated in the peripheral cornea or at the edge of the treatment zone. ${ }^{7-11}$ A retrospective series has described a frequency of $0.97 \%{ }^{7}$ while a prospective study of 117 eyes evaluating the complication rate of CXL found that sterile infiltrates occurred in $7.6 \%$ of eyes and with a stromal scar developing in $2.9 \% .6$ In our series, the rate of sterile keratitis at our institution is $2.7 \%$ (4 in 148 cases).

A prospective study looking specifically at the complications of CXL found that no parameter was predictive for developing sterile infiltrates. ${ }^{6}$ From our literature review, we could find no study that highlighted risk factors for the development of this sterile keratitis. Hypotheses for possible aetiologies have included alterations in antigenicity that occur in native proteins after CXL, leading to patients recognizing the proteins as non-self and mounting immune responses, ${ }^{7}$ and enhanced cell-mediated immunity to staphylococcal antigen deposition in areas of static tear pooling beneath the bandage contact lens. ${ }^{11}$

In our patients, the fact that all cases occurred on a single list did recommend the possibility of the cases being infective. UVA radiation is bactericidal, but infectious keratitis after CXL can and does occur. ${ }^{13-15}$ However, we feel that this is most unlikely. We found from our investigations that the instruments and solutions used for CXL came from different batches, the gram stains and cultures were negative, and significant improvements in the patients' signs and symptoms only occurred with the initiation of topical steroids. Despite developing sterile infiltrates, the prognosis for these patients is good. The unaided visual acuities improved in three of the four patients and the maximum Ks reduced in three out of four patients.

The clue to the possible etiology of the keratitis comes from the morphology of the patients' corneas. We found that a thinnest corneal pachymetry of $<425 \mu \mathrm{m}$ and a

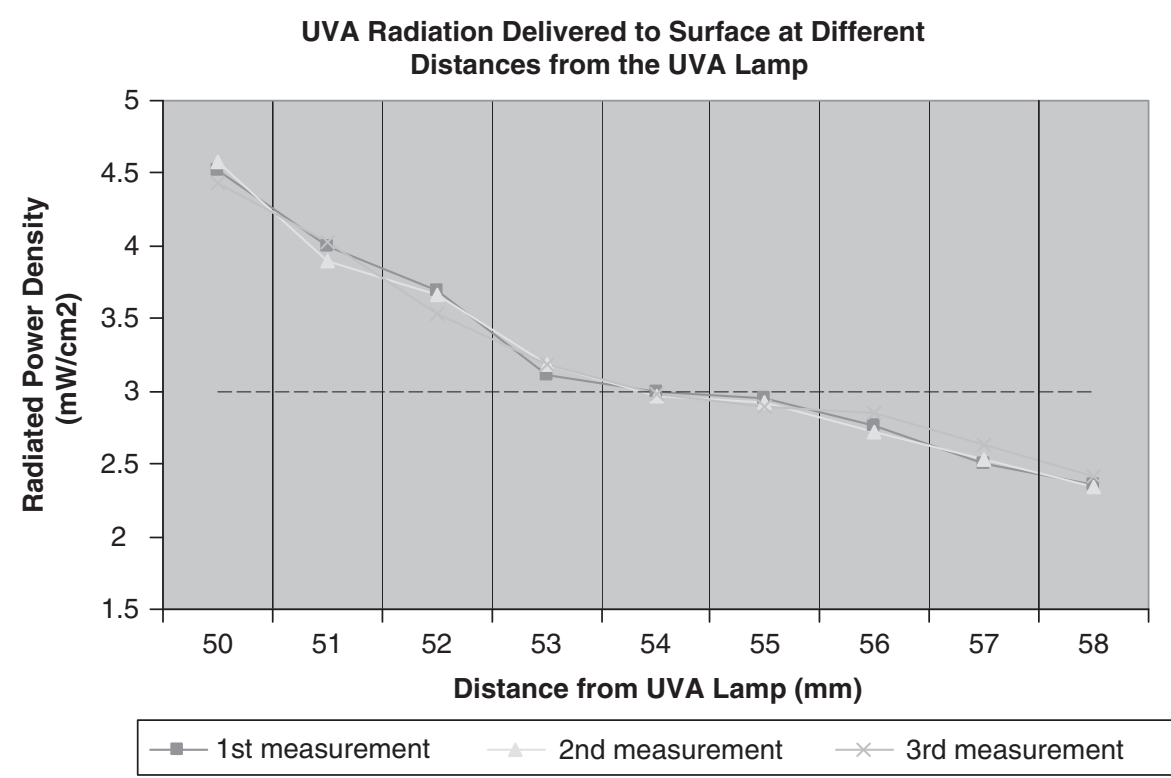

Figure 2 UVA irradiance when the UVA lamp is set at different distances. 
maximum corneal curvature of $>60 \mathrm{D}$ to be significant risk factors for the development of sterile keratitis, and when these risk factors were combined, the risk of sterile keratitis was significantly higher. The cause of the sterile infiltrate in these cases could be endothelial toxicity.

In routine practice, the standard protocol of CXL includes, for safety purposes a minimum corneal thickness of $400 \mu \mathrm{m}$ after removal of corneal epithelium because a cytotoxic level of UVA irradiation is reached in human corneas with a stromal thickness of less than $400 \mu \mathrm{m} .{ }^{16}$ In-vitro experiments using endothelial cell cultures from porcine corneas have revealed that an abrupt cytotoxic threshold irradiance level was found at $0.35 \mathrm{~mW} / \mathrm{cm}^{2}$ after combined treatment with riboflavin plus UVA irradiation. ${ }^{17}$ This is equivalent to a standard surface UVA dose of $3 \mathrm{~mW} / \mathrm{cm}^{2}$ when the corneal thickness is $400 \mu \mathrm{m} .{ }^{18}$

With our method of CXL, the corneal thickness is preserved as corneal epithelium is not debrided.

However, the all four patients who developed a corneal infiltrate had a corneal pachymetry at the apical cone of $<436 \mu \mathrm{m}$ and a thinnest corneal pachymetry of $<425 \mu \mathrm{m}$ (Table 1). In fact, one of the four cases had a corneal thickness of $378 \mu \mathrm{m}$ at the apex of the cone putting him below the safe threshold in this area despite having seemingly satisfactory central corneal pachymetries. However, treatment was carried out after a discussion of the risks because of the continued progression of his keratoconus since there is no other current treatment that can halt the progression of keratoconus. We believe that thinner corneas allowed a greater exposure of the corneal endothelium to UVA radiation leading to endothelial toxicity, which was then a trigger for the immune response and the sterile infiltrates. Further evidence comes from the fact that the sterile infiltrates tended to occur in the thinnest area of the cornea. One patient even developed an endothelial plaque, although we are uncertain as to why the infiltrates otherwise tended to appear at anterior or mid-stromal depth.

In relation to the relevance of maximum corneal curvature of the cornea, we hypothesize that eyes with steeper corneal curvatures are at a greater risk of developing sterile infiltrates because the ocular surface of eyes with steeper corneal curvatures retain riboflavin drops less well. Wollensak et $a l^{19}$ reported that dextranriboflavin solution used in the standard CXL procedure forms a stable $70-\mu \mathrm{m}$ pre-corneal film after $1 \mathrm{~min}$ and had a breakup time of $22 \mathrm{~min}$ in human donor corneas, which reduced the UVA irradiance in stroma at a depth of $400 \mu \mathrm{m}$ from 0.68 to $0.21 \mathrm{~mW} / \mathrm{cm}^{2}$ to shield the corneal endothelium from UVA. Steeper corneal curvatures could thus lead to a thinner pre-corneal riboflavindextran pre-corneal film and reduced shielding of the corneal endothelium and the thresholds for toxicity to be exceeded. However, despite how appealing this hypothesis might be, were unable to find a significant difference between the endothelial cell densities preoperatively and at 3 month.

Could an alternative hypothesis be keratocyte toxicity? It is well-known that CXL leads to the apoptosis of keratocytes and studies with confocal microscopy have confirmed this effect down to a depth of $320 \mu \mathrm{m}$. By 3 months, there is a recovery in the population keratocytes with an increased density of extracellular matrix compatible with a subclinical, microscopically detectable haze. ${ }^{20}$ Clinically significant haze with a reduction of vision can develop between the second and the third month after CXL and is resistant to topical steroids. ${ }^{21}$ This permanent haze can occur in $8.6 \%$ and it has been found that patients who developed this have been found to have higher $K$-values at the apex of keratoconus and thinner corneas pre-operatively. ${ }^{22}$ However, the characteristics, time course, pathophysiology, and evolution of this haze is clearly different to the sterile infiltrates that we are describing.

We found that the UVA irradiance on a treated corneal surface could vary greatly if the working distance of the UVA lamp was changed. If the VEGA C.B.M. X-Linker was brought closer to an irradiated surface by $2 \mathrm{~mm}$, the surface irradiance increased to between 3.5 and $3.7 \mathrm{~mW} / \mathrm{cm}^{2}$. As the treatment doses that are used in current regimes are already close to the toxic endothelial doses for corneal thicknesses of $400 \mu \mathrm{m}$, even a small increase in irradiation from these changes in working distance would result in these thresholds for endothelial toxicity of $0.36 \mathrm{~mW} / \mathrm{cm}^{2}$ being exceeded in patients with thinner corneas. In fact, the locations of the corneal infiltrates in our affected patients were at the thinnest parts of their cornea.

Given the retrospective nature of the enquiry, we were unable to discount if the UVA lamp had been systematically set at too close a working distance during the CXL procedures. The UVA lamp may have been placed too close to the patients' eyes for the duration of the CXL list leading to a larger dose of UVA being administered to each patient for the entirety of the CXL list. Any movement of the patient during surgery could lead to a change in the working distance. In the situation where the working distance is inadvertently reduced, the size of the treatment beam would be rendered smaller on the patient's cornea and the treatment would be, paradoxically, more easy to keep centered well within the patient's corneal limbus.

In conclusion, individuals with thinner corneas and higher corneal curvatures appear to be at higher risk of developing a sterile infiltrate. Our findings point towards endothelial toxicity being a strong possible cause of sterile infiltrates. The irradiance from a UVA lamp for 
corneal collagen cross-linking is vulnerable to small fluctuations of working distance. Although three of the four patients who developed these infiltrates experienced an improvement in their vision, extra care is recommended for patients with thinner corneas and steeper corneal curvatures who present with progressive keratoconus. Further research into the efficacy of alternative protocols in such patients would be beneficial.

\section{Summary}

What was known before

- Sterile keratitis can develop following uncomplicated corneal collagen cross-linking.

- To date, no clear predisposing factor for sterile keratitis has been reported.

\section{What this study adds}

- Patients with thinner and steeper corneas are at an increased risk of developing sterile keratitis.

- The visual outcomes, despite sterile keratitis, are good.

- Minor changes in the working distance of the lamp can lead to significant changes in the UVA irradiance on a cornea.

\section{Conflict of interest}

The authors declare no conflict of interest.

\section{References}

1 Krachmer JH, Feder RS, Belin MW. Keratoconus and related noninflammatory corneal thinning disorders. Surv Ophthalmol 1984; 28(4): 293-322.

2 Wollensak G, Spoerl E, Seiler T. Riboflavin/ultravioleta-induced collagen crosslinking for the treatment of keratoconus. Am J Ophthalmol 2003; 135(5): 620-627.

3 Caporossi A, Baiocchi S, Mazzotta C, Traversi C, Caporossi T. Parasurgical therapy for keratoconus by riboflavin-ultraviolet type A rays induced cross-linking of corneal collagen: preliminary refractive results in an Italian study. J Cataract Refract Surg 2006; 32(5): 837-845.

4 Wittig-Silva C, Whiting M, Lamoureux E, Lindsay RG, Sullilvan LK, Snibson GR. A randomized controlled trial of corneal collagen cross-linking in progressive keratoconus: preliminary results. J Refract Surg 2008; 24(7): S720-S725.

5 Koller T, Iseli HP, Hafezi F, Vinciguerra P, Seiler T. Scheimpflug imaging of corneas after collagen cross-linking. Cornea 2009; 28(5): 510-515.

6 Koller T, Mrochen M, Seiler T. Complication and failure rates after corneal crosslinking. J Cataract Refract Surg 2009; 35(8): 1358-1362.
7 Ghanem RC, Netto MV, Ghanem VC, Santhiago MR, Wilson SE. Peripheral sterile corneal ring infiltrate after riboflavin-UVA collagen cross linking in keratoconus. Cornea 2012; 31: 702-705.

8 Camesasca FI, Vinciguerra P, Seiler T. Bilateral ring-shaped intrastromal opacities after corneal cross-linking for keratoconus. J Refract Surg 2011; 27(12): 913-915.

9 Mangioris GF, Papadopoulou DN, Balidis MO, Poulas JL, Papadopoulos NT, Seiler T. Corneal infiltrates after corneal collagen cross-linking. J Refract Surg 2010; 26(8): 609-611.

10 Koppen C, Bryghem JC, Gobin L, Tassignon MJ. Keratitis and corneal scarring after UVA/ribofloavin cross-linking for keratoconus. J Refract Surg 2009; 25(9): S819-S823.

11 Angunawela RI, Arnalich-Montiel F, Allan BDS. Peripheral sterile corneal infiltrates and melting after collagen crosslinking for keratoconus. J Cataract Refract Surg 2009; 35: 606-607.

12 Pollhammer M, Cursiefen C. Bacterial keratitis early after corneal crosslinking with riboflavin and ultraviolet-A. J Cataract Refract Surg 2009; 35: 588-589.

13 Zamora KV, Males JJ. Polymicrobial keratitis after a collagen crosslinking procedure with postoperative use of a contact lens: a case report. Cornea 2009; 28: 474-476.

14 Rama P, Di Matteo F, Matuska S, Paganoni G, Spinelli A. Acanthamoeba keratitis with perforation after corneal crosslinking and bandage contact lens use. J Cataract Refract Surg 2009; 35: 788-791.

15 Samaras KE, Lake DB. Corneal collagen cross linking (CXL): a review. Int Ophthalmol Clin 2010; 50(3): 89-100.

16 Wollensak G. Crosslinking treatment of progressive keratoconus: new hope. Curr Opin Ophthalmol 2006; 17(4): 356-360.

17 Wollensak G, Sporl E, Reber F, Pillunat L, Funk R. Corneal endothelial cytotoxicity of riboflavin/UVA treatment in vitro. Ophthalmic Res 2003; 35(6): 324-328.

18 Wollensak G, Spoerl E, Wilsch M, Seiler T. Endothelial cell damage after riboflavin-ultraviolet-A treatment in the rabbit. J Cataract Refract Surg 2003; 29(9): 1786-1790.

19 Wollensak G, Aurich H, Wirbelauer C, Sel S. Significance of the riboflavin film in corneal collagen crosslinking. J Cataract Refract Surg 2010; 36(1): 114-120.

20 Mazzotta C, Balestrazzi A, Traversi C, Baiocchi S, Caporossi T, Tommasi $C$ et al. Treatment of progressive keratoconus by riboflavin-UVA-induced cross-linking of corneal collagen: ultrastructural analysis by Heidelberg Retinal Tomograph II in vivo confocal microscopy in humans. Cornea 2007; 26(4): 390-397.

21 Mazzotta C, Balestrazzi A, Baiocchi S, Traversi C, Caporossi A. Stromal haze after combined riboflavin_UVA corneal collagen cross-linking in keratoconus: in vivo confocal microscopi evaluation. Clin Experement Ophthalmol 2007; 35: 580-582.

22 Raiskup F, Hoyer A, Spoerl E. Permanent corneal haze after riboflavin-UVA-induced cross-linking in keratoconus. J Refract Surg 2009; 25: S824-S828. 